\title{
The effect of chromatic surround upon generalization along an angularity dimension in pigeons
}

MARTIN R. BARON and ELAINE L. BRESNAHAN, 1 Kent State University, Kent, Ohio 44240

Generalization gradients along the angularity dimension were examined following training to a vertical white line. During training and test the surround color remained green or black. Half of the Ss received nondifferential training; half received differential training to presence vs absence of the line. Differential training produced steeper gradients than nondiffer ential training; surround color and type of training interacted to influence gradient slope.

Newman \& Baron (1965) found a steep generalization gradient along the angularity dimension following differential training to presence vs absence of a vertical white line on a green surround; a horizontal "gradient" was obtained following similar training to a vertical white line on a green surround vs a red surround (no line). This evidence suggests (Baron, 1965) that for pigeons, attention is selective and that chroma is attended more than white line presence. ${ }^{2}$

One implication of the above interpreta. tion is that the gradient along the angularity dimension would be steeper if the line were green (on a white surround) than if the line were white (on a green surround). Employing a differential training procedure, Baron \& Vacek (1967) obtained results consistent with this expectation. Their findings may be interpreted to mean that there was greater attention to the green line on the white surround than to the white line on the green surround. One may question, however, whether the chroma of the green line increased attention to the line, or whether chroma in the green surround decreased attention to the white line. The present study was designed to examine the latter possibility. During training and generalization test the surround color remained either green or black. Half of the Ss were provided nondifferential training to the vertical white line; half were provided differential training to its presence vs absence.

\section{METHOD}

The Ss were 48 experimentally naive homing pigeons obtained from a local supplier.

Three identical Skinner-type pigeon boxes described in Newman \& Baron (1965) were utilized. An overhead jewelled GE 47 bulb illuminated each box.
The stimuli, projected on the key by a display cell, consisted of a green $(578 \mathrm{~m} \mu)$ light and a white line $(1 / 4 \times 1$ in. $)$ tilted at one of five angular orientations varied in 30-deg steps from a position $60 \mathrm{deg}$ counterclockwise to 60 deg clockwise from the vertical. The surround could be made black by switching off the light source for the $578-\mathrm{m} \mu$ filter.

White noise was continuously presented via a speaker mounted in the ceiling of each box.

On Day 1, Ss were unsystematically assigned to one of four treatment groups, and were magazine and key-peck trained. The stimulus key contained a white vertical line on a green surround for Group D-G (differentially trained) and Group ND-G (nondifferentially trained) and on a black surround for Groups D-B and ND-B. Each pecking response at the key provided $3 \mathrm{sec}$ of food reinforcement. Each $\mathrm{S}$ was allowed to make 100 continuously reinforced responses, 50 on Day 1, and an additional 50 on Day 2.

On Days 3 through 12, all Ss were gradually shifted to a VI schedule in which two reinforcements were randomly programmed during each 55 -sec presentation of St. For Groups D-G and D-B, $3055-\mathrm{sec}$ St presentations were alternated with $15-\mathrm{sec}$ $S-$ presentations in an ABBA BAAB sequence. Responses to $S$ - (a green or black key with no line present) were never reinforced. For Groups ND-G and ND-B the St remained unchanged for $3055-\mathrm{sec}$ periods.

On Day 13, Ss were given a warm-up session immediately followed by a generalization test. For Groups D-G and D-B warm-up consisted of six alternations of $\mathrm{S}+$ and $S-(45 \mathrm{sec}$ each) with two randomly spaced reinforcements provided during each St presentation. Ss in Groups ND-G and ND-B received reinforcement on the same schedule during six $45-\sec$ periods with $\mathrm{S}+$ on the key. The test consisted of 10 presertations ( $45 \mathrm{sec}$ each) of the five test angles in a counterbalanced sequence; no reinforcements were provided. There were no blackout periods during training or generalization test.

\section{RESULTS}

The total number of responses made by the four groups to each of the test stimuli (analyzed by an analysis of variance) are shown in Fig. 1.

The gradient for Group D-G appears somewhat steeper than that for
Group ND-G, and the gradient for Group D-B appears steeper than that for Group ND-B. Consistent with these observations, the interaction of Type of Training (differential-nondifferential) by Test Stimuli was significant $(F=5.14$, df $=4 / 176$, $\mathrm{p}<.01$ )

The gradient for Group D-B (differentially trained with a black surround) is steeper than that for Group D-G. In contrast, the gradient for Group ND-G (nondifferentially trained with the green surround) is steeper than that for Group ND-B. This interaction (Type of Training by Surround Color by Test Stimuli) was significant $(F=2.65, \quad \mathrm{df}=4 / 176$, $\mathrm{p}<.05)$. These data suggest that the chromatic surround decreased attention to the vertical white line during differential training but increased attention to the line during nondifferential training

The interaction between surround color and test stimuli was separately examined for each of the two types of training (differential and nondifferential). In neither case was there a significant effect. Thus, neither the attention-decreasing effect of the chromatic surround in differentia training nor the attention-increasing effect of the chromatic surround in nondifferential training was significant when examined separately.

Because a sloped gradient along the angularity dimension was obtained with Group ND-G (nondifferentially trained with a white line on a green surround) and a flat "gradient" had been obtained (Newman \& Baron, 1965) with a group trained under somewhat similar conditions, an exact replication of Groups ND-G and ND-B was performed. This replication produced

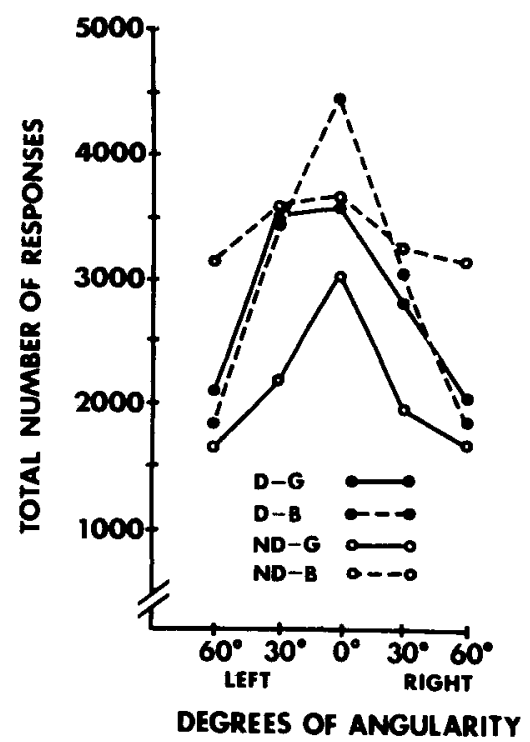

Fig. 1. Number of responses to test stimuli for four groups. 
results comparable to those described above. A sloped gradient was obtained with the green surround, and the gradient was steeper with the green than with the black surround. The gradients, however, were not significantly different $(F=1.34, \quad$ df $=4 / 83$, $\mathrm{p}>.05)$

\section{DISCUSSION}

Consistent with results commonly re ported in the literature (e.g., Newman \& Baron, 1965), gradients along the angularity dimension were steeper following differential than nondifferential training. In the Newman and Baron study, however, a flat "gradient" was obtained following nondif ferential training to a white vertical line on a green surround while similar training in the present experiment and in the replication produced sloped gradients. There were a number of procedural differences between the two studies; which one accounts for the difference in slope is not known, however.

Evidence reported in the present paper regarding the effect of chromatic surround is difficult to reconcile with the suggestion of Baron (1965) and those of Mackintosh (1965) and Sutherland (1964). According to their formulations, attention to one aspect or dimension of a complex stimulus would be expected to produce inattention to others. Thus it was anticipated, assuming that chroma was more salient than a white vertical line, that attention to a chromatic surround might produce inat tention to a line and its angularity during either nondifferential or differential training. The results of this study show that the effect of chroma in the surround upon attention to a line is different with nondifferential and differential training.

\section{REFERENCES}

BARON M. R. The stimulus, stimulus control, and stimulus generalization. In D. I. Mostofsky (Ed.), Stimulus generalization. Stanford, Calif: Stanford University Press, 1965.

BARON, M. R., \& VACEK, C. T. Generalization gradients along wavelength and angularity dimensions in pigeons following two differential training procedures. Psychonomic Science, $1967,9,423-424$.

MACKINTOSH, N. J. Selective attention in animal discrimination leaming. Psychological Bulletin, 1965, 64, 124-150.

NEWMAN, F. L., \& BARON, M. R. Stimulus generalization along the dimension of angularity: A comparison of training procedures. Journal of Comparative \& Physiological Psychology, 1965, 60, 59-63.

SUTHF RLAND, N. S. The leaming of discrimination by animals. Endeavour, 1964, 23, 148-152.

\section{NOTES}

1. This paper is based upon a thesis submitted by the second author in partial fulfillment of the requirements for the MA degree at Kent State University, 1966.

2. This interpretation is consistent with the selective attention theories of Mackintosh (1965) and Sutherland (1964).

3. This replication was performed by 1 ynda $S$. Doll.

\section{Development and maintenance of FR escape with titrated reinforcement'}

JAMSHID KHALILI and CARL. CHENEY, Utah State University, Logan, Utah 84321

High fixed-ratio grid escape behavior was developed and maintained when a titrated reinforcement procedure was used in the acquisition of a lower ratio. FR20 developed in one session was maintained as long as 170 sessions. Escape responding remained consistent when the schedule was raised to FR 40, FR60, and even FR80.

Few studies investigating escape procedures with rats report success in acquisition or maintenance of consistent behavior with continuous grid shock. Many Es attribute this failure to the methods of shock application and the different shock generators and devices (Dinsmoor, 1967; Hendry \& Hendry, 1963). Although it is not denied that a better procedure for shock application is necessary, we believe that a great deal of this problem can be eliminated by the use of modified acquisition training procedures.

Recently a procedure for acquisition training of ratio and interval schedules of negative reinforcement has been reported (Khalili \& Daley, 1968). In this procedure the ratio size (or mean interval length) does not vary during the acquisition period as is common in intermittent schedule training. Briefly the procedure is as follows: After a session of continuous reinforcement (CRF) of escape responding with a 30-sec time-out (T.O.) from shock, the schedule is set at the desired ratio or interval. In the following session all responses, other than the terminal response which produces a $30-\mathrm{sec}$ T.O., will produce a smaller amount of reinforcement (T.O. from shock). The short time-out periods for each response are called titrated reinforcers (TRs), and the TR value is decreased in steps until it reaches 0 . A typical set of TR values might be TR $10 \mathrm{sec}$, TR5 sec, TR $1 \mathrm{sec}$, and TR0 sec. TR0 sec is the same as the primary schedule. This procedure of stepwise decreasing CRF for all responses, except the terminal one, is referred to as a titrated negative reinforcement procedure.

The purpose of our experiment was to investigate some parameters of acquisition and maintenance of fixed-ratio schedules as well as transitional effects of ratio increases.

\section{METHOD}

Six male hooded Long-Evans rats were used. All animals had continuous access to food and water in their home cages.
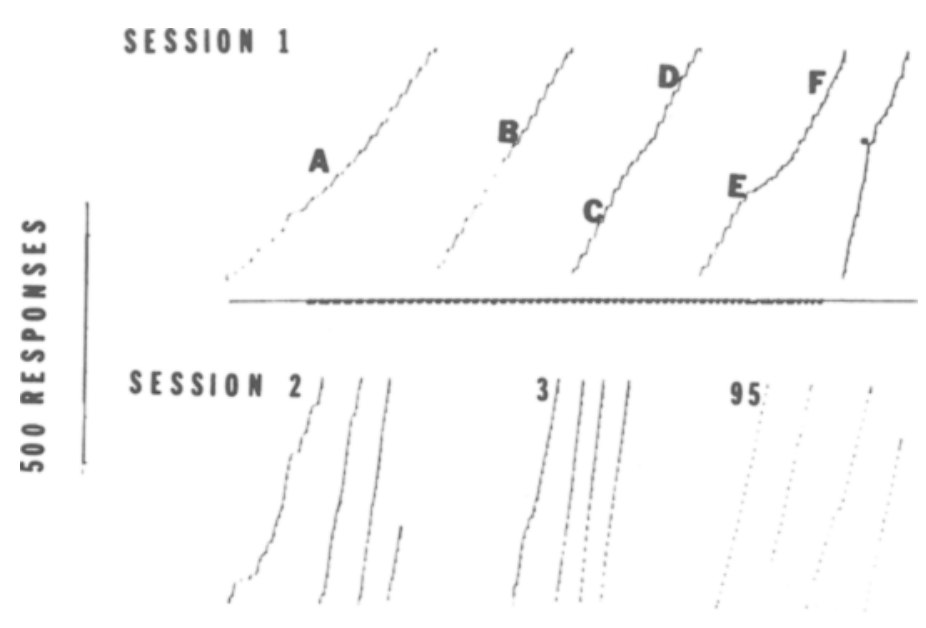

95

3OMINUTES

Fig. 1. Representative cumulative records of FR20 escape acquisition with decreasing TRs within a session (top) and maintenance for 95 sessions. Knees indicated at a. 